\title{
Uso y conocimiento de la fauna silvestre de la comunidad q'eqchi' de Santa Lucía Lachuá, Alta Verapaz, Guatemala: un abordaje etnozoológico
}

\author{
Use and knowledge of the wild fauna of Santa Lucía Lachuá \\ Q'eqchi'community in Alta Verapaz, Guatemala: an ethnozoological \\ approach
}

\author{
Emily Pineda-Posadas ${ }^{1 *}$, Usi’j Bá-Velásquez¹, Julio Morales-Álvarez¹, José Pellecer-González¹, \\ Melanie Ortiz-De León ${ }^{1}$, Ramiro Tox-Cao (QEPD) ${ }^{2}$
}

'Escuela de Biología, Facultad de Ciencias Químicas y Farmacia, Universidad de San Carlos de Guatemala, Guatemala,

${ }^{2}$ Santa Lucía Lachuá, Alta Verapaz, Guatemala

* Autor a quien se dirige la correspondencia: emi.pin.22@gmail.com

Recibido: 5 de diciembre de 2020/ Aceptado: 16 de abril de 2021

Resumen

— sta investigación tuvo como objetivo sistematizar información etnozoológica de los usos y elementos del conocimiento Clocal de la comunidad q'eqchi' de Santa Lucía Lachuá, Alta Verapaz, en relación al sexo y grupo etario de los habitantes. La metodología consistió en realizar entrevistas semiestructuradas, con un muestreo por conveniencia a 41 personas, 21 hombres y 20 mujeres, entre adultos y adultos mayores, a quienes se les mostraron fotografías de especies de vertebrados para su reconocimiento. A partir del Índice de valor de uso (IVU), se calculó el número de especies reconocidas por las personas entrevistadas. Los mamíferos fueron de los vertebrados más reconocidos y aprovechados por la comunidad, seguido por las aves, reptiles y anfibios y por último los peces. Las especies con mayor valor de uso por la comunidad q'eqchi' son Odocoileus virginanus (0.4759), Dasypus novemcinctus (0.4747) Cuniculus paca (0.4735), Amazona farinosa guatemalensis (0.4462) Iguana iguana (0.4747), y Parachromis friedrichsthalii (0.4700). No se observó una diferencia en el conocimiento adquirido por sexo y grupo etario. Se resalta la importancia de continuar con los estudios etnozoológicos en la ecorregión Lachuá y otros territorios del país para fortalecer los procesos de gestión y conservación de la diversidad biocultural.

Palabras clave: Conocimiento local, fauna silvestre, grupo etario, valor de uso, etnobiología

\begin{abstract}
This research was carried out with the goal of systematizing ethnozoological information regarding the uses and elements of 1 local knowledge of the Q'eqchi' village of Santa Lucía Lachuá, Alta Verapaz in relation to sex and age class. The methodology consisted of conducting semi-structured surveys using images for the recognition of vertebrates` species and following the convenience sampling method to 41 people, split between 21 men and 20 women, and separated into two age groups, adults and the elderly. We calculated the use value index (UVI) of each recognized species by the respondents. Mammals were the most recognized and showing more uses in the sample. Followed by birds, reptiles and amphibians, and fishes. The results among the species with the highest use value in our Q'eqchi' sample are Odocoileus virginanus (0.4759), Dasypus novemcinctus (0.4747), Cuniculus paca (0.4735), Amazona farinosa guatemalensis (0.4462) Iguana iguana (0.4747), and Parachromis friedrichsthalli $(0.4700)$. We did not observe any difference in knowledge between sex and age groupings. We highlight the importance to continue ethnozoological studies in the ecorregión Lachuá and other territories of the country to strengthen the processes of management and conservation of biocultural diversity.
\end{abstract}

Keywords: Local knowledge, wild fauna, sex, age group, use value, ethnobiology

C Autor(es). Ciencia, Sociales y Humanidades, es editada por la Universidad de San Carlos de Guatemala, bajo licencia Creative Commons Atribución-NoComercial-Compartir Igual 4.0 (https://creativecommons.org/licenses/ by-nc-sa/4.0/legalcode). El contenido de esta publicación es responsabilidad de su(s) autor(es). 


\section{Introducción}

Los q'eqchi' se llaman así mismos aj ral ch'och 'b "hijos de la tierra". Para ellos, es más importante "el estar situado en un lugar" que el "ser-esencia" del castellano "ser" (Wilson, 1999), pues es la tierra el elemento de identidad, producción, recolección y caza de especies silvestres, en la construcción del conocimiento local (Hatse \& De Ceuster, 2001). Esta forma de entender el conocimiento es estudiada por la etnozoología, la cual es la disciplina que estudia el conocimiento local de la fauna y de las relaciones culturales entre las comunidades humanas y entre la fauna de su ambiente (Hunn, 2011). La relación de los grupos humanos con la naturaleza crea diversidad de relaciones y asociaciones con la vida silvestre, que constituyen un legado histórico y cultural en la evolución humana, permitiéndole desarrollar conocimiento. Este conocimiento etnozoológico abarca la percepción, el uso y el manejo de la fauna silvestre (Gutiérrez-Santillán et al., 2017).

En Mesoamérica, los pueblos indígenas se posicionan como actores, usuarios y constructores de prácticas y conocimientos locales que se han formado a través de procesos socioculturales, de observación e interacción con los entornos naturales (Guerra et al., 2010; Monroy et al., 2011). De tal manera que los pueblos indígenas le han dado usos específicos a la fauna silvestre para satisfacer sus necesidades, como alimento, intercambio, recreación o para fines medicinales. Pero en otra dimensión la fauna es parte esencial de las significaciones, de lo subjetivo, lo simbólico y místico-religioso (García-Flores et al., 2017; Santos-Fita et al., 2012).

El pueblo q'eqchi' considera a la tierra un espacio sagrado e indispensable para la estructura de sus comunidades, y su forma de relacionarse con la tierra la consideran correcta o "verdadera" (Estrada, 2006). Esta cosmovisión, así como el conjunto de conocimientos y significaciones, son el resultado de experiencias que mediante procesos de transmisión generacional de enseñanza-aprendizaje han construido un proceso de prácticas que tratan a la naturaleza de manera respetuosa. Las comunidades q'eqchi' se han establecido en gran parte del territorio guatemalteco, siendo Alta Verapaz uno de los departamentos con la mayor cantidad de su población (Estrada, 2006). Santa Lucía Lachuá es una comunidad q'eqchi' que forma parte de la ecorregión Lachuá en Alta Verapaz, y cuenta con una alta diversidad florística y faunística
(Avendaño Mendoza, 2001; Escuela de Biología de la Facultad de Ciencias Químicas y Farmacia, de la Universidad de San Carlos de Guatemala [USAC], 2004; Hernández, 2004; Hermes \& Rosales-Meda, 2011) que ha contribuido al desarrollo de prácticas de agricultura y cacería que sostienen sus medios de vida (González, 2004).

Las relaciones entre las personas de Santa Lucía Lachuá y la fauna local resultan de importancia por el valor que tiene el conocimiento local en la conservación de la diversidad biológica de la ecorregión, por lo que es necesario el retomar estudios etnobiológicos (y etnozoológicos) en el área. Sin embargo, los estudios etnobiológicos se han realizado desde un enfoque conceptual, con intereses en la conservación a diferentes escalas, de modo que es importante continuar con la sistematización del conocimiento local que promueva la valorización del mismo y que aporte en el diseño de estrategias de conservación y las prácticas en la comunidad. Desde esta perspectiva se desarrolla el presente estudio, con el propósito de sistematizar el conocimiento etnozoológico entre hombres y mujeres, adultos y adultos de la tercera edad de la comunidad q'eqchi' de Santa Lucía Lachuá, Cobán, Alta Verapaz.

\section{El problema}

El conocimiento local está íntimamente ligado a la cosmovisión de los pueblos y este ha cambiado y adaptado a los diferentes momentos históricos que ha enfrentado el pueblo q'eqchi'. Se observa entonces, deterioro del conocimiento local como resultado de un proceso histórico relacionado con las estructuras sociales, políticas e institucionales que han sostenido por años los sistemas locales de gestión y apropiación de recursos naturales. Esta erosión del conocimiento está ligada a cambios de uso del suelo, pérdida de idiomas locales, aculturación, escolarización y cambios más allá del contexto local como la industrialización, la migración, la integración en los mercados y los procesos de homogeneización sociocultural ligados a la globalización (Gómez-Baggethun, 2009).

Es imposible abordar este proceso de erosión del conocimiento local, si no se parte de una perspectiva histórica, la cual tiene un punto de ruptura durante la colonización española (siglo XVI), donde se da la imposición de valores y prácticas occidentales y ocurre la destrucción y prohibición de los elementos de la cosmovisión, como prácticas y rituales (Hatse \& De Ceuster, 2001). A finales del siglo XIX y principios 
$\mathrm{XX}$, el latifundismo incorpora adaptaciones a los rituales agrícolas y tergiversa las significaciones de conceptos importantes como el Tzuul Taq'a (Señor de los cerros y valles) para el pueblo q'eqchi'. Otra época histórica importante es la del conflicto armado interno, que tuvo rasgos marcadamente étnicos (Wilson, 1999), donde hubo persecución selectiva de ancianos y líderes, dejando a las comunidades sin orientación. Se estima que en total entre 90 y 100 aldeas fueron destruidas en Alta Verapaz (Wilson, 1999). Durante la represión militar, docenas de comunidades en el área de "Salawim" de la región de Lachuá fueron completamente destruidas y miles de sus habitantes asesinados (Flores, 2002).

La agenda conservacionista también ha impactado en la cosmovisión y conocimiento del pueblo q'eqchi' durante las décadas de los años 80 y 90, a pesar del conocimiento científico reconocido de los pueblos originarios de Mesoamérica. Los conservacionistas han idealizado las selvas como sitios de alta biodiversidad, enmarcando la sostenibilidad como una relación antagonista entre el campo y el bosque (Grandia, 2018), lo que representa concepciones separadas culturalmente acerca del significado y uso que tiene el bosque entre las personas q'eqchi' y los conservacionistas (Grandia, 2018). La noción de áreas protegidas para los q'eqchi' se traduce mal ajustado como ilb'il ch'och que significa "tierra vigilada", lo que denota una asombrosa negación o ignorancia de los valores espirituales del pueblo q'eqchi' para el bosque, ejemplo de un racismo institucional que invade las instituciones gubernamentales en Guatemala que descalifican el conocimiento y prácticas locales (Grandia, 2018).

Existe otro problema cuando se aborda el tema del conocimiento local de los pueblos originarios, y es la imposición e influencia de las iglesias evangélicas, la transferencia tecnológica y los "proyectos de desarrollo". Estos factores han incidido fuertemente en la conciencia, la identidad y conocimientos tradicionales de los pueblos indígenas (Hatse \& De Ceuster, 2001; Hernández-Freeman, 2009; Wilson, 1999).

La importancia del rescate y valoración del conocimiento local es impostergable por varias razones: (a) El corpus de conocimiento en sí mismo, (b) las opciones que este conocimiento aportan para el manejo de la vida silvestre, (c) la valoración de la cultura de los pueblos originarios como alternativa de adaptación y resiliencia civilizatoria, y (d) reconocimiento y conservación del patrimonio cultural de Guatemala. Por lo que es importante sistematizar el conocimiento local de comunidades q'eqchi' que cuentan con una riqueza histórica de manejo y conservación de la naturaleza, en este caso, de la comunidad q'eqchi' de Santa Lucía Lachuá, junto al área protegida Parque Nacional Laguna Lachuá. A través de este estudio, se pretende dar un primer paso a nuevos paradigmas en la conservación de la fauna considerando no solo las perspectivas de la academia, sino también el de las comunidades locales que tienen su propia forma de conservar.

\section{Materiales y métodos}

\section{Ubicación geográfica del estudio}

La comunidad de Santa Lucía Lachuá está ubicada en el municipio de Cobán, a 140 kilómetros de la cabecera departamental de Alta Verapaz. Es una de las 44 comunidades que se encuentran en la zona de influencia del Parque Nacional Laguna Lachuá (PNLL) (coordenadas $15^{\circ} 46^{\prime}$ latitud norte y $90^{\circ} 45^{\prime}$ longitud oeste) (Consejo Nacional de Áreas Protegidas [CONAP] et al., 2004; Escuela de Biología USAC, 2004) (Figura 1).

\section{Figura 1}

Localización de la Aldea Santa Lucía Lachuá, municipio de Cobán, departamento de Alta Verapaz, Guatemala

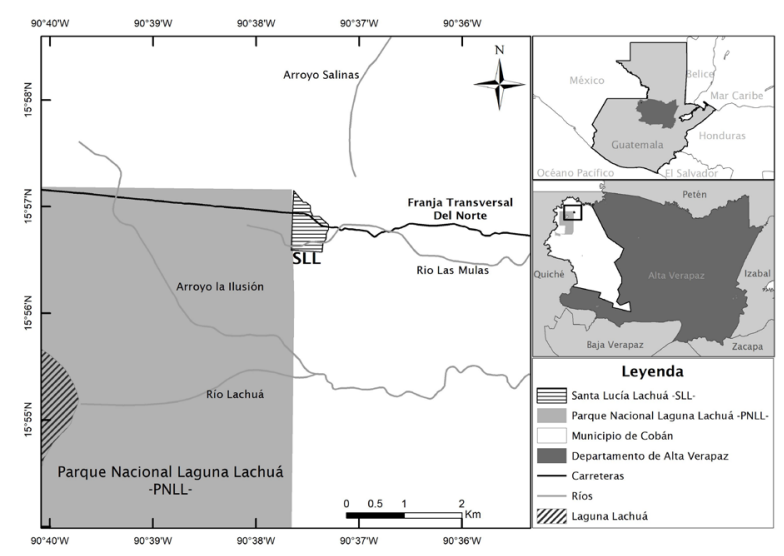

La comunidad es atravesada por la carretera asfaltada Franja Transversal del Norte, la cual, al llegar al límite entre la aldea y el Parque, esta se interrumpe por un camino de terracería que ingresa al área protegida. En la parte sur es atravesada por el río Las Mulas.

El área de estudio se ubica en un relieve plano a $200 \mathrm{msnm}$, precipitación anual de 3,330 mm, 91.2\% 
de humedad relativa y $25.3^{\circ} \mathrm{C}$ de temperatura (CONAP et al., 2004). Forma parte de la ecorregión Lachuá, un sitio de importancia RAMSAR (Convención de Humedales de Importancia Internacional) y uno de los últimos remanentes boscosos del arco húmedo (Wendt, 1987). Alrededor del Parque Nacional Laguna Lachuá (PNLL) se encuentra un mosaico de remanentes de selva, dominado por pastizales para ganado al noroeste y más heterogéneo al noreste (Escuela de Biología USAC, 2004). También se discriminan al menos 8 tipos de vegetación en función de su uso, entre ellas cultivo de milpa, potreros, guamiles, bosques de cardamomo, bosque latifoliado y en la última década ha aumentado el uso de monocultivo de palma africana (Elaeis guineensis), teniendo efectos en la riqueza de vertebrados de la zona (Escuela de Biología USAC, 2004; Rojas \& Avendaño, 2018).

En la ecorregión habitan cerca del $20 \%$ de las especies de mamíferos reportadas para Guatemala, entre ellas jaguar (Panthera onca), puma (Puma concolor), tapir (Tapirus bairdii) y tumboarmado (Cabassous centralis) (Hermes, 2004; Ruano et al., 2019; Pellecer et al., 2019). Especies de reptiles como Crocodylus moreletii, elápidos, vipéridos y colúbridos; anfibios como Rhinophrynus dorsalis y Rhinella marinus; y 177 especies de aves, incluyendo 29 migratorias (Avendaño Mendoza, 2001; CONAP et al., 2004). Además, en el área se pueden encontrar especies de árboles como el tamarindo (Dialium guianense), ceiba (Ceiba pentandra), san juan (Vochysia hondurensis), caoba (Swietenia macrophyla), y cedro (Cedrela odorata) (CONAP et al., 2004).

\section{Contexto histórico y actual de Santa Lucía Lachuá}

De acuerdo a CONAP y colaboradores (2011), la población humana actual de la Ecorregión Lachuá está compuesta por 18,500 habitantes; con 2,410 familias y cerca del $59 \%$ tiene una edad inferior a 15 años y el $40 \%$ se encuentra entre 16 y los 45 años. En Santa Lucía Lachuá, los registros de los primeros asentamientos en la zona datan de 1979 como resultado de la demanda de tierras y la guerra interna (CONAP, 2003). La mayoría de la población pertenece a la comunidad q'eqchi', aproximadamente con 400 adultos y familias compuestas entre 5 y 8 integrantes (A. Xo, comunicación personal, 2018).

Las personas de la comunidad se dedican a actividades comerciales y agrícolas de maíz, frijol, arroz, sandía y cardamomo, principalmente (CONAP, 2003). El uso de tierras para estas actividades ha generado conflictos asociados a la pertenencia de tierras desde los años 90, así como deficiencias en los servicios básicos hacia los hogares de los pobladores y enfermedades provocadas por contaminación del recurso hídrico (Escuela de Biología USAC, 2004; Marqués-Valdés, 2014).

\section{Recolección de datos y temporalidad del estudio}

El estudio se inició como un ejercicio académico en el contexto de un "Curso Taller" impulsado y diseñado por el docente Claudio Méndez y otros profesores de la Escuela de Biología en la Estación Biológica Santa Lucía Lachuá en marzo del año 2018.

El muestreo se realizó utilizando el criterio de muestreo por conveniencia (Otzen \& Manterola 2017), que permitió seleccionar aquellos casos accesibles que aceptaron ser incluidos después de familiarizarnos con su contexto cultural y su idioma. La colaboración de dos personas de la comunidad: Don Ramiro Tox (QEPD) y Héctor Tox, permitió establecer el vínculo comunicativo traduciendo en algunos casos del español al q'eqchi', para lograr una comunicación a profundidad entre ambas partes. Cabe mencionar que varias personas no aceptaron ser entrevistadas o no se encontraban en sus domicilios.

Los participantes en el estudio fueron personas mayores de edad (18 años en adelante) de la comunidad q'eqchi' de Santa Lucía Lachuá, divididas en 20 mujeres y 21 hombres, que representan el $10.3 \%$ de la población de la comunidad (compuesta por 400 adultos) (A. Xo y J. J. Cucúl, comunicación personal, 2020). Se realizaron recorridos aleatorios por la comunidad visitando cada una de las familias, en donde se pidió el consentimiento de las personas que fueran mayores de edad para participar en el estudio.

\section{Técnicas e instrumentos}

Se aplicó una entrevista semiestructurada y conversaciones abiertas individuales para recabar la información (Russell, 2006; Vela, 2001). La entrevista contenía preguntas referentes a la etnozoología de la comunidad y abordó dos temas principales respecto a la fauna silvestre local: (a) los usos que las personas les dan como alimento, comercialización, ornamento, medicina o mascota, y (b) los conocimientos que tie- 
nen sobre las especies locales respecto a su hábitat, patrones de actividad (si las especies se avistan en la noche o en el día), avistamiento de crías y simbolismo.

Como técnica complementaria y para facilitar el reconocimiento de fauna, a cada persona entrevistada, se le mostraron láminas con fotografías de 100 especies locales y no locales de cuatro grupos de vertebrados: mamíferos, aves, reptiles-anfibios y peces (Dos Santos, 2009, p. 260). Se anotó el número de la (s) fotografía(s) que las personas reconocían o demostraron conocer y, a partir de ello, se profundizó en las preguntas de la entrevista, obteniendo como resultado información más específica sobre determinada especie.

Previo al levantado de la información, y como prueba piloto para mejorar el instrumento antes de presentarlo a las demás personas (Osorio, 1998), se conformó un grupo focal de personas de la comunidad, conformado por guías locales y colabores de investigaciones en el área a quienes se les compartió la entrevista y las fotografías. Esta dinámica permitió obtener información valiosa para entender el contexto de la población de Santa Lucía Lachuá.

\section{Procesamiento y análisis de la información}

La información obtenida se sistematizó en una base de datos, en la hoja de cálculo Microsoft Excel (versión 2013). Se realizó un conteo de las especies reconocidas por cada entrevistado y se calculó el promedio de estos datos para determinar las especies reconocidas por grupo de vertebrados. Para establecer la diferencia entre los usos y conocimientos de la fauna de acuerdo al sexo y grupo etario de los entrevistados, se contabilizó la cantidad de personas (según sexo y grupo etario) que indicaron los siguientes usos: alimento, comercial, medicinal, ornamental o mascota. De igual manera se realizó con los conocimientos sobre aspectos de la fauna.

Asimismo, se calculó el índice de valor de uso (IVU) para cada especie mencionada (Alves Barbosa et al., 2010; De la Ossa-Lacayo \& De la Ossa, 2012), el cual permitió identificar las especies con mayor importancia cultural en el aprovechamiento por parte de la comunidad (Rodas-Trejo et al., 2014), mediante la fórmula: $\mathrm{IVU}=\sum \mathrm{U} / \mathrm{n}$, en donde: IVU = índice de valor de uso de la especie; $\mathrm{U}=$ número de usos mencionados para la especie por informante; y $\mathrm{n}=$ número de entrevistados.

Por último, se describieron los usos y valores simbólicos de algunas de las especies reconocidas, así como el nombre de las especies en idioma q'eqchi'.

\section{Ética en la investigación}

Para desarrollar la presente investigación se trabajó a partir del Código de Ética de la Sociedad Latinoamericana de Etnobiología (SOLAE, 2016) siguiendo las siguientes normas: (1) la responsabilidad de los involucrados de realizar una consulta para la obtención del consentimiento libre e informado de los líderes comunitarios y las familias de la comunidad de Santa Lucía Lachuá, reconociendo y asumiendo los sistemas normativos locales, (2) el derecho de los entrevistados para preservar su intimidad y (3) el derecho a ser consultados, explicándoles la importancia y el impacto del tema.

Por lo anterior, en cada entrevista se explicó a cada familia visitada y a cada persona entrevistada, los propósitos y objetivos de la investigación, del anonimato de sus respuestas y el derecho de cada persona a no aceptar ser entrevistada o detener la misma en cualquier momento.

\section{Resultados}

Género y edades de los entrevistados en la comunidad q'eqchi' de Santa Lucía Lachuá

Se entrevistaron a 41 personas en total, distribuidas de acuerdo a su sexo en 21 hombres y 20 mujeres, y a su grupo etario en cuatro categorías diferentes (Tabla 1).

\section{Tabla 1}

Edades según el sexo de los entrevistados en la comunidad q'eqchi'de Santa Lucía Lachuá

\begin{tabular}{cccc}
\hline $\begin{array}{c}\text { Rango de } \\
\text { Edades }\end{array}$ & $\begin{array}{c}\text { Categoría de } \\
\text { edades }\end{array}$ & $\begin{array}{c}\text { Total } \\
\text { Mujeres }\end{array}$ & $\begin{array}{c}\text { Total } \\
\text { Hombres }\end{array}$ \\
\hline 18-26 años & Adulto Joven & 5 & 1 \\
$27-39$ años & Adulto C1 & 4 & 10 \\
$40-59$ años & Adulto C2 & 9 & 6 \\
$60>$ años & Adulto C3 & 2 & 4 \\
\hline
\end{tabular}

Cabe resaltar que, para la comunidad, la población adulta está dividida en dos: adultos (18-44 años) y adultos mayores ( $\geq 45$ años). La edad promedio de los entrevistados fue de 42 años, con una edad mínima de 18 años y una edad máxima de 70 años. 
Reconocimiento de especies por los entrevistados de la comunidad q'eqchi' de Santa Lucía Lachuá

Los entrevistados, tanto hombres como mujeres, mencionaron al menos una especie de los cuatro grupos de vertebrados. En total, reconocieron 68 especies y cada entrevistado reconoció entre 6 y 19 especies de mamíferos, 1-12 especies de aves, 2-18 especies de anfibios y reptiles y 1-19 especies de peces. Las entrevistas reportan a nivel de reconocimiento los siguientes promedios: mamíferos 11.5 especies, aves 6.9 especies, peces 6 especies y reptiles-anfibios 5.9 especies. De igual manera, hombres y mujeres mencionaron reconocer mayor cantidad de especies de mamíferos, en relación a los otros grupos de vertebrados (Figura 2).

\section{Figura 2}

Gráfica de especies de los cuatro grupos de mamíferos, mencionadas por los entrevistados. A. Mujeres y sus rangos etarios, $B$. Hombres y sus rangos etarios
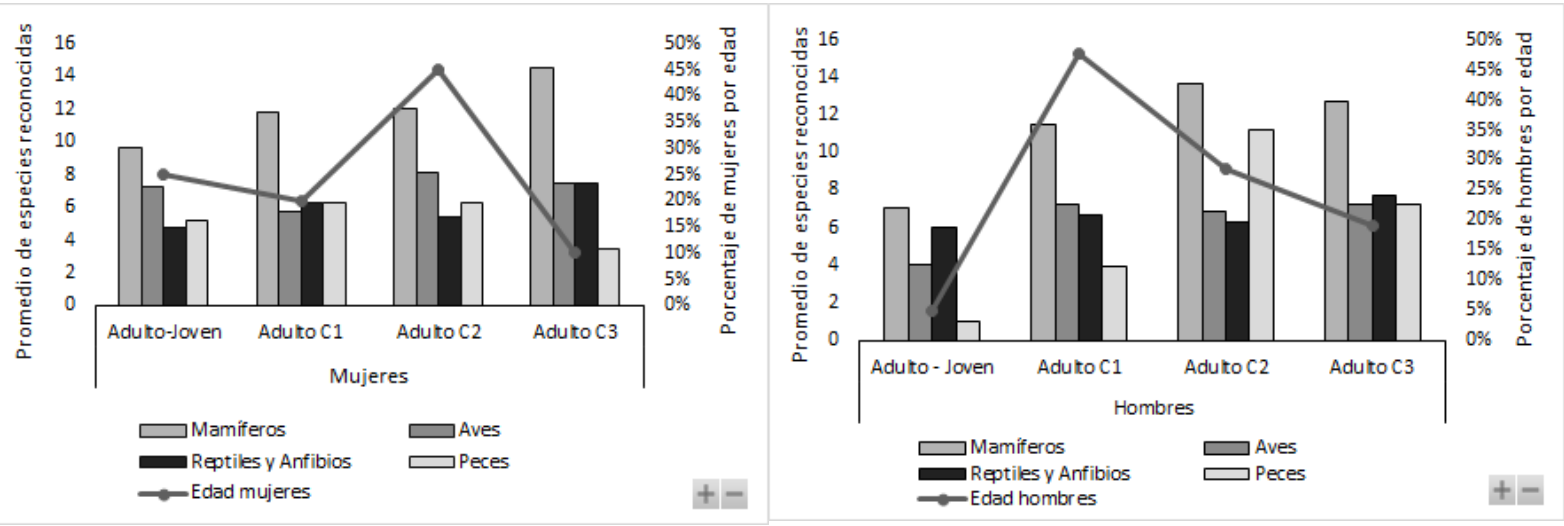

Todas las personas entrevistadas utilizan la fauna con fines alimentarios y la minoría para fines de ornato y mascota (Tabla 2).

Tabla 2

Usos de la fauna silvestre por sexo y grupo etario de los entrevistados de la comunidad q'eqchi' de Santa Lucía Lachuá

\begin{tabular}{|c|c|c|c|c|c|c|}
\hline \multirow{2}{*}{ Mujeres } & \multirow{2}{*}{ Hombres } & \multicolumn{5}{|c|}{ Usos } \\
\hline & & Alimento & Medicina & Ornamento & Comercio & Mascota \\
\hline Adulta & - & 12 & 5 & 3 & 10 & 2 \\
\hline Adulta mayor & - & 8 & 7 & 1 & 8 & 4 \\
\hline- & Adulto & 12 & 6 & 2 & 11 & 1 \\
\hline- & Adulto mayor & 9 & 8 & 2 & 8 & 1 \\
\hline \multicolumn{2}{|c|}{ Total mujeres } & 20 & 12 & 4 & 18 & 6 \\
\hline \multicolumn{2}{|c|}{ Total hombres } & 21 & 14 & 4 & 19 & 2 \\
\hline \multicolumn{2}{|c|}{ Total adultos } & 24 & 11 & 5 & 21 & 3 \\
\hline \multicolumn{2}{|c|}{ Total adultos mayores } & 17 & 15 & 3 & 16 & 5 \\
\hline & Total & 41 & 26 & 8 & 37 & 8 \\
\hline
\end{tabular}


De acuerdo al sexo y grupo etario, las personas reconocieron tener usos similares. Según el sexo, la mayoría de las mujeres y hombres afirmaron usar especies de fauna silvestre con fines alimentarios y de comercio, y les asignaron menor uso a fines de mascota y ornamento. El resultado es similar en adultos y adultos mayores, sin embargo, éstos últimos son quienes mayoritariamente utilizan la fauna para fines medicinales.

Por otro lado, la totalidad de los entrevistados afirmaron conocer aspectos de la fauna relacionados con el hábitat de las especies, y solo pocas personas conocían sobre los patrones de actividad (Tabla 3).

Tabla 3

Conocimientos de la fauna silvestre por sexo y grupo etario de los entrevistados de la comunidad q'eqchi' de Santa Lucía Lachuá

\begin{tabular}{|c|c|c|c|c|c|}
\hline \multirow[b]{2}{*}{ Mujeres } & \multirow[b]{2}{*}{ Hombres } & \multicolumn{4}{|c|}{ Conocimiento } \\
\hline & & Simbólico & Hábitat & Actividad & Crías \\
\hline Adulta & - & 10 & 12 & 1 & 9 \\
\hline Adulta mayor & - & 7 & 8 & 3 & 4 \\
\hline- & Adulto & 6 & 12 & 3 & 8 \\
\hline - & Adulto mayor & 9 & 9 & 3 & 4 \\
\hline \multicolumn{2}{|c|}{ Total mujeres } & 17 & 20 & 4 & 13 \\
\hline \multicolumn{2}{|c|}{ Total hombres } & 15 & 21 & 6 & 12 \\
\hline \multicolumn{2}{|c|}{ Total adultos } & 16 & 24 & 4 & 17 \\
\hline \multirow{2}{*}{\multicolumn{2}{|c|}{ Total adultos mayores }} & 16 & 17 & 6 & 8 \\
\hline & & 32 & 41 & 10 & 25 \\
\hline
\end{tabular}

Según el sexo, tanto hombres como mujeres comparten conocimientos sobre el hábitat de las especies, su valor simbólico y avistamiento de crías. De acuerdo al grupo etario, los adultos y adultos mayores comparten conocimientos sobre el valor simbólico de las especies, pero difieren en que la mayoría de adultos afirmaron conocer las crías de las especies y los adultos mayores los patrones de actividad de las especies.

Especies de fauna aprovechadas por los entrevistados de Santa Lucía Lachuá

Las personas entrevistadas aprovechan 63 especies de fauna, mayormente de mamíferos. De acuerdo al IVU de las especies, la mayoría de mamíferos presentaron valores altos del índice, siendo los mayores el venado cola blanca (Odocoileus virginianus), el armadillo de nueve bandas (Dasypus novemcinctus) y el tepezcuintle (Cuniculus paca). Seguido de reptiles como la iguana (Iguana iguana) y peces (mojarra paleta ó Vieja bifasciata, mojarra colorada o V. melanura y el guapote o Parachromis friedrichsthalii) (Figura 3, Tabla 4).
Para aves los valores de uso fueron menores, siendo el loro corona azul (Amazona farinosa guatemalae) el que mayor valor posee.

Los entrevistados reconocieron tener usos variados para especies de mamíferos como el venado, el cabro de monte (Mazama temama), el armadillo de nueve bandas y el tacuazín (Didelphis virginiana) (Tabla 5), lo cual es consistente con la Figura 2, en donde se muestran que estas especies presentaron mayores valores de uso.

Para las demás especies, afirmaron tener un uso particular, ya sea como alimento, medicina, mascota, comercio, y un valor simbólico de importancia cultural en la comunidad. Tal es el caso de búhos y colibríes, cuyo valor simbólico es el de ser mensajeros, mientras que los psitácidos (loros) fueron las únicas especies utilizadas como mascotas. Además, a las especies de peces, reptiles y anfibios les asignan usos alimenticios y comerciales, principalmente. 


\section{Figura 3}

Índice de valor de uso (IVU) para las especies reconocidas por las personas entrevistadas de Santa Lucía Lachuá
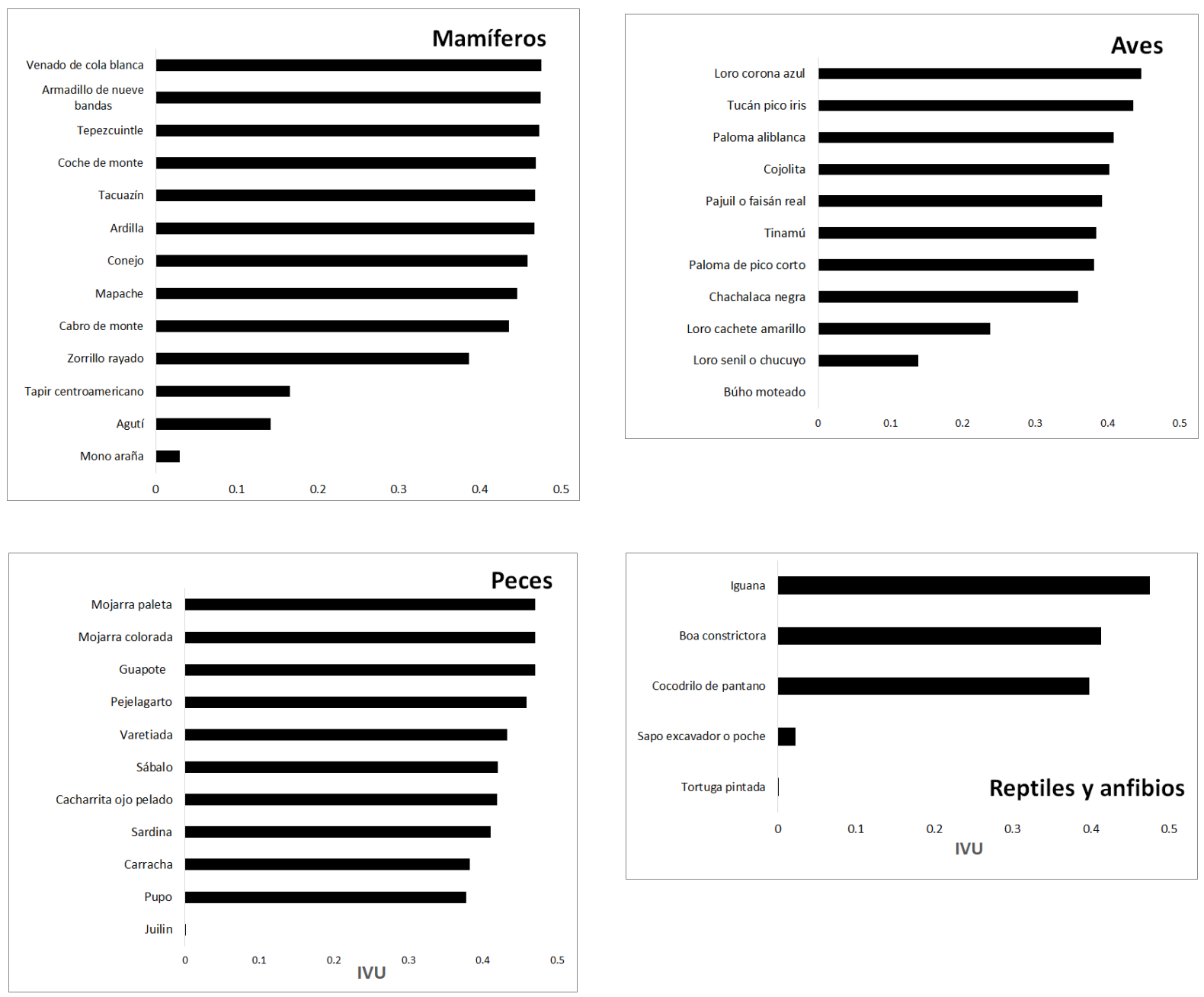


\section{Tabla 4}

Nombres cientificos y comunes de las especies aprovechadas por los entrevistados y sus correspondientes indices de valor de uso

\begin{tabular}{|c|c|c|c|c|}
\hline Grupo & Especie/ Nombre común & IVU & Especie / Nombre común & IVU \\
\hline \multirow{11}{*}{ Mamíferos } & $\begin{array}{l}\text { Odocoileus virginianus / Venado cola } \\
\text { blanca }\end{array}$ & 0.4759 & Mustela frenata / Comadreja & 0.3819 \\
\hline & $\begin{array}{l}\text { Dasypus novemcinctus / Armadillo nueve } \\
\text { bandas }\end{array}$ & 0.4747 & $\begin{array}{l}\text { Cabassous centralis / Armadillo cola } \\
\text { desnuda }\end{array}$ & 0.3647 \\
\hline & Cuniculus paca / Tepezcuintle & 0.4735 & Nasua narica / Pizote & 0.3486 \\
\hline & Tayassu tajacu / Pecarí de collar & 0.4688 & Tapirus bairdii / Tapir & 0.1660 \\
\hline & Didelphis virginiana / Tacuazín & 0.4681 & Dasyprocta punctata / Agutí & 0.1422 \\
\hline & Sciurus yucatanensis / Ardilla & 0.4676 & Ateles geoffroyi / Mono araña & 0.030 \\
\hline & Tayassu pecari / Pecarí labiado & 0.4652 & Philander opossum / Zarigueya gris & 0.0220 \\
\hline & Sylvilagus floridanus / Conejo & 0.4593 & Alouatta pigra / Mono aullador & 0.0012 \\
\hline & Conepatus semistriatus / Zorrillo de franjas & 0.4569 & Rattus norvegicus / Rata común & 0.0012 \\
\hline & Procyon lotor / Mapache & 0.4461 & Herpailurus yagouaroundi / Yaguarundi & 0.0006 \\
\hline & $\begin{array}{l}\text { Mazama temama / Cabro de monte } \\
\text { Mephitis macroura / Zorrillo rayado }\end{array}$ & $\begin{array}{l}0.4361 \\
0.3867\end{array}$ & Chiroptera / Murciélagos & 0.0006 \\
\hline \multirow{8}{*}{ Aves } & $\begin{array}{l}\text { Amazona farinosa guatemalensis / Loro } \\
\text { corona azul }\end{array}$ & 0.4462 & Penelopina nigra / Chachalaca negra & 0.3593 \\
\hline & Ramphastos sulfuratus / Tucán pico iris & 0.4355 & $\begin{array}{c}\text { Aulacorhynchus prasinus / Tucán } \\
\text { esmeralda }\end{array}$ & 0.3206 \\
\hline & Zenaida asiatica / Paloma aliblanca & 0.4081 & $\begin{array}{l}\text { Amazona autumnalis / Loro cachete } \\
\text { amarillo }\end{array}$ & 0.2374 \\
\hline & Penelope purpurascens / Cojolita & 0.4021 & Pionus senilis / Loro senil o chucuyo & 0.1386 \\
\hline & Crax rubra / Pajuil o faisán & 0.3926 & Momotus momota / Motmot coroniazul & 0.0006 \\
\hline & Tinamus major / Tinamú & 0.3843 & Dendrocygna autumnalis / Pijije & 0.0006 \\
\hline & Columba nigrirostris / Paloma & 0.3807 & Tytonidae / Rapaces nocturnas & 0.0006 \\
\hline & Trochilidae / Colibríes & 0.3736 & & \\
\hline \multirow{3}{*}{$\begin{array}{l}\text { Anfibios y } \\
\text { reptiles }\end{array}$} & Iguana iguana / Iguana verde & 0.4747 & $\begin{array}{l}\text { Rhinophrynus dorsalis / Sapo excava- } \\
\text { dor opoche }\end{array}$ & 0.0226 \\
\hline & Boa constrictor / Boa & 0.4128 & Trachemys scripta / Tortuga pintada & 0.0006 \\
\hline & Crocodylus moreletii / Cocodrilo, lagarto & 0.3980 & & \\
\hline \multirow{10}{*}{ Peces } & Parachromis friedrichsthalii / Guapote & 0.4700 & Thorichthys affinis / Cacharra & 0.3962 \\
\hline & Vieja bifasciata / Mojarra colorada & 0.4700 & Brycon guatemalensis / Machaca & 0.3950 \\
\hline & Vieja melanura / Mojarra paleta & 0.4700 & Thorichthys pasionis / Carracha & 0.3819 \\
\hline & Atractosteus tropicus / Pejelagarto & 0.4587 & Poecilia mexicana / Pupo & 0.3778 \\
\hline & Oscura heterospila / Pintia & 0.4468 & Maskaheros argenteus / Pozolera & 0.3742 \\
\hline & Cribroheros robertsoni / Varetiada & 0.4325 & Ictalurus meridionalis / Jolote & 0.3730 \\
\hline & Megalops atlanticus / Sábalo & 0.4194 & Eugerres mexicanus / Pichincha & 0.3724 \\
\hline & Thorichthys meeki / Cacharrita & 0.4188 & Dorosoma anale / Arenque & 0.3712 \\
\hline & Potamarius nelsoni / Curuco & 0.4123 & Astyanax aeneus / Pepesca & 0.3694 \\
\hline & Dorosoma petenense / Sardina & 0.4105 & Rhamdia guatemalensis / Juilin & 0.0012 \\
\hline
\end{tabular}

Nota: *IVU: Índice de valor de uso 
Tabla 5

Usos y valor simbólico de las especies reconocidas por las personas entrevistadas de la comunidad q'eqchi' de Santa Lucía Lachuá

\begin{tabular}{|c|c|c|c|c|}
\hline Grupo & Especie & $\begin{array}{l}\text { Nombre } \\
\text { común }\end{array}$ & $\begin{array}{l}\text { Nombre } \\
\text { q'eqchi' }\end{array}$ & Usos / Valor simbólico \\
\hline & $\begin{array}{l}\text { Odocoileus } \\
\text { virginianus }\end{array}$ & $\begin{array}{l}\text { Venado de cola } \\
\text { blanca }\end{array}$ & Kej & $\begin{array}{l}\text {-Uso alimenticio: consumen su carne } \\
\text {-Uso ornamental: la piel es utilizada para } \\
\text { decorar sillas }\end{array}$ \\
\hline & Mazama temama & Cabro de monte & kaxlan yuk & $\begin{array}{l}\text {-Uso alimenticio: consumen su carne con } \\
\text { un alto valor nutricional } \\
\text {-Uso ornamental: el cráneo y las astas } \\
\text { del animal son usados como objetos de } \\
\text { adorno para las casas (Figura 4) }\end{array}$ \\
\hline & Cuniculus paca & Tepezcuintle & Halaw & -Uso alimenticio: consumen su carne \\
\hline \multirow[t]{5}{*}{ Mamíferos } & $\begin{array}{l}\text { Dasypus } \\
\text { novemcinctus }\end{array}$ & $\begin{array}{l}\text { Armadillo de } \\
\text { nueve bandas }\end{array}$ & i’boy & $\begin{array}{l}\text {-Uso medicinal: el caparazón del armadi- } \\
\text { llo lo utilizan para curar y la carne la con- } \\
\text { sumen para tratar problemas respiratorios } \\
\text {-Uso alimenticio: consumen su carne } \\
\text {-Uso ornamental: el caparazón y la cola } \\
\text { los usan como adorno (Figura 4) }\end{array}$ \\
\hline & Mustela frenata & Comadreja & saqb' in & $\begin{array}{l}\text {-Uso medicinal: utilizan a la comadreja } \\
\text { para tratar sustos (Chilin) }\end{array}$ \\
\hline & Didelphis & Tacuazín & Uch & $\begin{array}{l}\text {-Uso alimenticio: consumen la carne de } \\
\text { tacuazín } \\
\text {-Uso medicinal: la manteca del tacuazín } \\
\text { la utilizan para tratar el asma y los } \\
\text { calambres }\end{array}$ \\
\hline & $\begin{array}{c}\text { Conepatus } \\
\text { semistriatus, } \\
\text { Mephitis macroura }\end{array}$ & Zorrillo & par & $\begin{array}{l}\text {-Uso medicinal: usado para tratar el dolor } \\
\text { de huesos }\end{array}$ \\
\hline & Chiroptera & Murciélagos & zotz $^{\prime}$ & $\begin{array}{l}\text {-Valor simbólico: emisarios de malas } \\
\text { noticias }\end{array}$ \\
\hline
\end{tabular}

\begin{tabular}{|c|c|c|c|c|}
\hline \multirow{3}{*}{ Aves } & Ciccaba virgata & $\begin{array}{l}\text { Tecolote o búho } \\
\text { moteado }\end{array}$ & tucu, warom & $\begin{array}{l}\text {-Valor simbólico: anunciantes de enfer- } \\
\text { medad o muerte }\end{array}$ \\
\hline & Trochilidae & Colibríes & tz'unun & $\begin{array}{l}\text {-Valor simbólico: anunciantes de buenas } \\
\text { noticias }\end{array}$ \\
\hline & $\begin{array}{l}\text { Amazona } \\
\text { autumnalis } \\
\text { A. farinosa } \\
\text { guatemalae }\end{array}$ & Loro & chocho' & $\begin{array}{l}\text { Uso como mascota: los psitácidos son } \\
\text { utilizados como mascotas por algunas } \\
\text { personas }\end{array}$ \\
\hline $\begin{array}{l}\text { Reptiles y } \\
\text { anfibios }\end{array}$ & Iguana iguana & Iguana verde & Iwaan & -Uso alimenticio: consumen su carne \\
\hline
\end{tabular}




\section{Discusión}

Etnozoología de la población q'eqchi' de Santa Lucía Lachuá

La transmisión del conocimiento de la diversidad biológica, de prácticas de manejo y otros componentes ecológicos, en comunidades originarias está acompañada de la sucesión de responsabilidades, de padres a hijos, o de la sucesión de cargos cívicos o religiosos (Millán et al., 2016), por lo que se esperaría que las generaciones de entrevistados de Santa Lucía Lachuá tuvieran el mismo conocimiento sobre fauna local. Esto se refleja en las mismas proporciones de adultos y adultos mayores en la cantidad de vertebrados que reconocen, los usos que les dan y otras características como hábitat y patrones de actividad de las especies (Tablas 2 y 3 ). El uso y conocimiento compartido de la fauna entre los entrevistados se debe a la inclusión que padres y abuelos permiten a las nuevas generaciones, en la que jóvenes adquieren conocimientos derivados de hechos y prácticas que posiblemente apliquen en su edad adulta (Millán et al., 2016). Por ello, se ve un aumento relativo y paulatino en el conocimiento sobre la fauna, proporcional a la edad del entrevistado, pero no se descarta que esta tendencia esté sesgada por el número de personas por rango etario que fueron entrevistadas (Figura 2).

Se observaron diferencias de usos y conocimientos de fauna entre grupos etarios. Respecto a los usos de fauna, en la categoría medicinal se percibió una indiferencia de los más jóvenes de usar animales para curar enfermedades (Tabla 2). De hecho, los adultos mayores entre ellos un curandero de la comunidad, aunque con reservas, dio la mayor información sobre el uso de vertebrados para estos fines. No se han encontrado estudios con sustento científico de laboratorio que confirme o niegue la efectividad de este tipo de medicinas, aunque los entrevistados aseguran que sí ha funcionado en los casos particulares donde se han utilizado. A pesar de que el curanderismo es una práctica utilizada en la ecorregión (CONAP, 2003), pareciera que la transmisión de este conocimiento a las nuevas generaciones se está perdiendo o son menos propensos a utilizarlo, ya que cuentan con otras opciones para curar enfermedades, a través de los centros de salud y farmacias que hay actualmente en la comunidad. Por la naturaleza de los datos obtenidos y del análisis realizado, no se puede asegurar la relevancia e importancia que es para las nuevas dinámicas de la comunidad el saber de fauna con fines medicinales y en su lugar suplir por medicina occidental.

El conocimiento de usos medicinales e incluso simbólicos, recae en la importancia que implica dentro de la comunidad, el saber de estos aspectos a temprana edad. Koster y colaboradores (2016) consideran que, si la adquisición del conocimiento etnobiológico entre adolescentes y adultos es vital para el bienestar familiar y consecutivamente, en el bienestar comunitario, el conocimiento de la población más joven, llega a igualar al de sus mayores. Sin embargo, falta profundizar las causas de esta posible pérdida del conocimiento local y cuál es la dinámica familiar en cuanto a la transmisión de conocimientos ancestrales y actuales, si hay o no un choque generacional respecto al conocimiento que jóvenes y adultos están dispuestos a compartir y seguir transmitiendo, y si hay otras influencias, como las instituciones educativas y los medios de comunicación, que interfieren y modifican estas dinámicas comunitarias. Se sugiere a futuro ampliar los grupos de entrevistados tomando en cuenta las generaciones emergentes que serían los niños y adolescentes, y como su proceso de aprendizaje integra o no estos conocimientos locales de fauna y sus usos.

Las diferencias en las respuestas de las categorías de uso menos utilizadas (ornamental y mascota), también puede estar sesgada a la actividad familiar de los padres o abuelos, ya que un entrevistado mencionó que guardó algunas partes de los animales que cazaba para adornar su hogar, aunque no especificó qué especie. Sin embargo, no todos quienes respondieron que tenían adornos de vertebrados o que llegaron a tener mascotas, admitieron ser cazadores ni especificaron el medio de obtención. En cuanto a otros conocimientos, además del uso, se encontró que los adultos conocen más sobre el hábitat de las especies y el avistamiento de crías, mientras que los adultos mayores conocen más sobre los patrones de actividad de las especies (Tabla 3). Ambos grupos reconocen el valor simbólico de estos vertebrados dentro de su comunidad. Un estudio reciente identifica que la mayoría de cazadores que encuestaron se encontraban en edades de 23 a 43 años (Ruano et al., 2019), lo que coincide con los conocimientos que poseen los entrevistados de este estudio según las categorías etarias establecidas. Pero, no se puede concluir la relación directa entre sus conocimientos, y las actividades que realizan en su comunidad, como lo es la cacería. Se sugiere realizar un estudio tomando en cuenta estas relaciones de oficio y profesión de los entrevistados y el grado de conocimiento sobre la fauna local. 
En cuanto al conocimiento y uso de fauna silvestre entre hombres y mujeres, diferentes estudios han descrito similitudes y diferencias entre sexos en la manera de conocer, utilizar y conceptualizar los recursos, visibilizando roles de género (Caniago \& Siebert, 1998; Vázquez-García, 2007; Viu et al., 2010). Sin embargo, esta división de roles depende de las circunstancias locales (Ladio, 2020). El enfoque de género en la cultura maya q'eqchi' considera la complementariedad entre hombre y mujer, es decir que, a pesar de la división de actividades en el seno familiar, ambos se complementan (Donato et al., 2007). Esto se refleja en los resultados obtenidos, en donde tanto hombres como mujeres conocen y utilizan la fauna de manera similar (Tablas 2 y 3), debido a la participación de la mujer q'eqchi' en actividades de recolección, comercialización (y en ocasiones de cacería no necesariamente de subsistencia), que le han permitido tener una estrecha relación con la naturaleza y conocer y utilizar aspectos de la fauna (como patrones de actividad, reproducción y hábitat de las especies) al igual que los hombres. Asimismo, se observó una comunicación homogénea de lo que se aprende sobre la fauna dentro de su territorio, no solo en la misma familia, sino a nivel comunitario, lo cual enfatiza la importancia de la mujer q'eqchi' como encargada de conservar la cultura y transmitir los conocimientos en el seno familiar (Caballero, 2013).

\section{Uso y conocimiento q'eqchi' sobre Mamíferos}

Los usos que los entrevistados tienen hacia la fauna son similares a otros estudios etnozoológicos en el Neotrópico (Cortés-Gregorio et al., 2013; Hernández-López et al., 2012; Racero-Casarrubia et al., 2008; Tlapaya \& Gallina, 2010), en donde los mamíferos constituyen el grupo más aprovechado para fines alimenticios, ya que son una de las principales fuentes de proteína de la población (Pérez \& Ojasti, 1996). Este es el caso de mamíferos grandes como el venado $(O$. virginianus) y el pecarí (Tayassu pecari), y especies de caza menor como el armadillo (D. novemcinctus) y el conejo (Sylvilagus floridanus) (Figura 3). Existe también preferencia por el sabor y consistencia de la carne, pues estas generan preferencias de consumo; por ejemplo, la carne del tepezcuintle es considerada un plato muy rico en la comunidad y se evidencia por su alto valor de uso (Morales, 1993). Estos resultados coinciden con un estudio previo llevado a cabo por Rosales y colaboradores (2010) en el periodo 2001-
2004 en la ecorregión Lachuá, en el cual reportan que las especies más cazadas son el tepezcuintle y el armadillo. En dicho estudio, los autores sugieren que la heterogeneidad del hábitat en la ecorregión podría afectar la disponibilidad de animales hacia los cazadores y por eso se han visto obligados a aumentar el esfuerzo de búsqueda y la distancia recorrida desde su comunidad hacia remanentes boscosos (Rosales et al., 2010). Además del uso alimentario, los mamíferos son el grupo de vertebrados con mayor cantidad de otros usos, como medicina, mascota, ventas y artesanías en comunidades originarias de México (Cortés-Gregorio et al., 2013; González-Bocanegra et al., 2011). Esto es similar a lo reportado en este estudio, en donde los entrevistados los utilizan como ornamento y como medicina (Figura 3, Tabla 4). Los mamíferos usados como ornamento, generalmente son cazados para alimento y una vez consumida su carne, los derivados (cráneos, pieles o colas) se utilizan como objetos artesanales (González-Bocanegra et al., 2011). Tal es el caso de algunas viviendas visitadas, en donde las familias tenían cráneos, astas de cabros y colas de armadillo (Figura 4).

También, los entrevistados reconocieron utilizar diferentes especies como medicina. Una de ellas es el armadillo (D. novemcinctus), que es utilizado para tratar diferentes síntomas de origen respiratorio a partir de infusiones del caparazón y la cola (López-Carrera et al., 2005; Tlapaya \& Gallina, 2010). Otras especies como el tacuazín y el zorrillo, son utilizados por su manteca o grasa para tratar numerosas enfermedades (López-Carrera et al., 2005; Cortés-Gregorio et al., 2013). Según el saber local, la comadreja (Mustela frenata) se consume para curar el "susto" o "chilin", que es una afección que ocurre cuando hay una interrupción súbita a una pareja durante el acto sexual y que genera síntomas de inapetencia, constipación y otros malestares que se menciona puede llevar a la muerte.

\section{Uso y conocimiento q'eqchi' sobre aves}

Las aves fueron el segundo grupo más aprovechado de acuerdo a los entrevistados, como alimento y mascota. Las especies de aves que presentaron mayor IVU (Tabla 4) son similares a reportes de comunidades indígenas de México y Venezuela, que afirman la preferencia alimenticia y como mascota por pajuiles (géneros Crax sp. y Penelope sp.), tucanes (Ramphastos) y loros (Amazona spp; Lugo-Morín, 2007; Racero-Casarrubia et al., 2008). El uso de animales 


\section{Figura 4}

Objetos derivados de mamíferos usados con fines ornamentales: A) Cola de armadillo, B) Cráneo y astas de cabro de monte

A

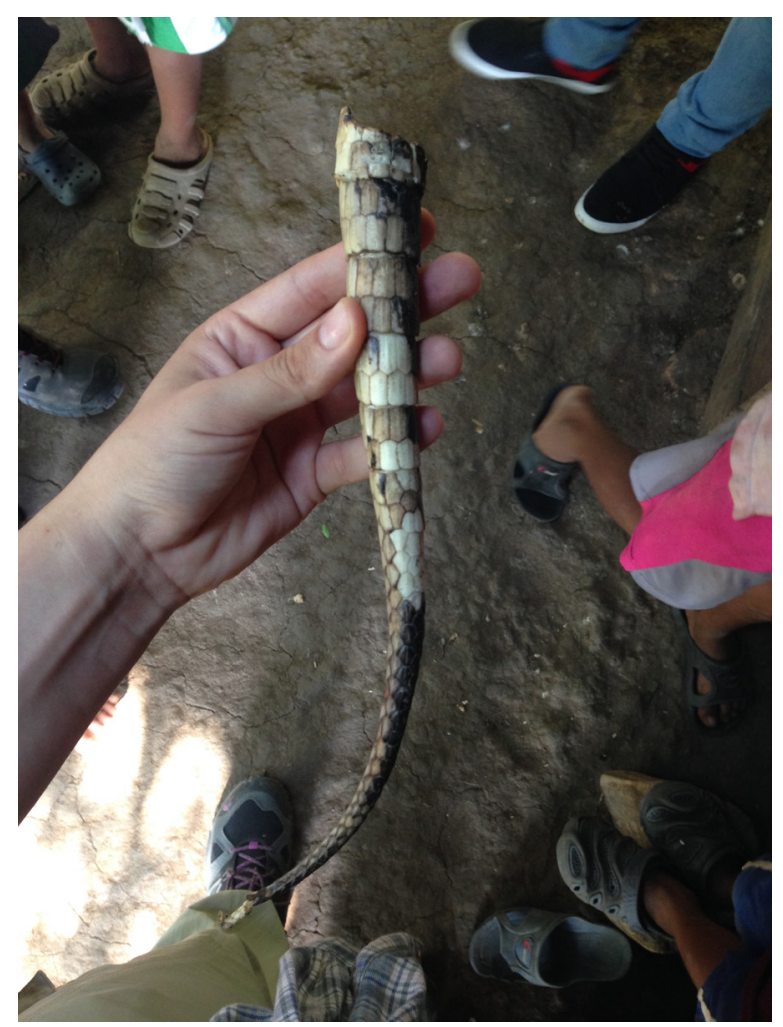

B

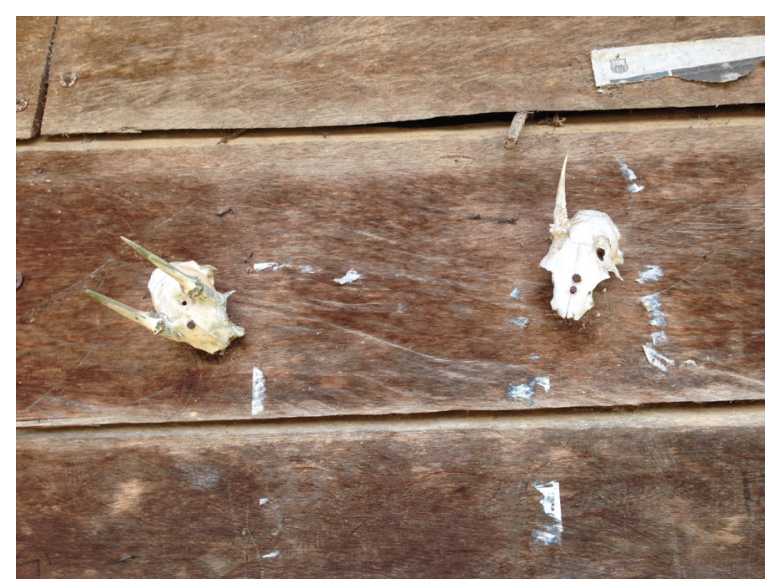

como mascota fue mencionado por pocas personas de la comunidad y se restringen a especies de psitácidos, lo que evidencia la influencia, control y vigilancia del área protegida PNLL (administrada por el Instituto Nacional de Bosques [INAB] y el CONAP), respecto al uso de animales silvestres. A su vez, la paloma (Columba, Zenaida) fueron de las aves con mayor valor de uso, debido a la cacería en la zona que ha sido documentada (Rosales et al., 2010).

Uso y conocimiento q'eqchi' sobre reptiles y anfibios

Los reptiles y anfibios fueron reconocidos por los entrevistados principalmente para usos con fines alimenticios y comerciales. La iguana (I. iguana) es una de las especies con mayor IVU en la comunidad (Figura 3), debido a que se utiliza como alimento y en algunos casos, al igual que la tortuga (Trachemys scripta) para comercio. Esto ha sido reportado para comunidades indígenas en Colombia y México (Cortés-Gregorio et al., 2013; Racero-Casarrubia et al., 2008). La mayoría de pobladores reconocen a las serpientes como símbolos de maleficios y anunciantes de enfermedades, al igual que a búhos y murciélagos (Tabla 5). En la comunidad, también existen relatos de una lagartija ligada a las palmas (Arecaceae) que provoca la atracción de rayos y la creencia muy generalizada que los geckos (especie introducida) son venenosos.

Los reptiles y anfibios están asociados a cuerpos de agua como riachuelos o pozas. Algunos entrevistados conocían el hábitat de algunas especies, como el sapo excavador (Rhinophrynus dorsalis), el cual se entierra y está asociado con la temporada de lluvias. Estos conocimientos locales sobre los hábitos de las especies son importantes para documentar su respuesta ante la fragmentación y los cambios del uso de la tierra que ocurren en el área (Stuart et al., 2008).

\section{Uso y conocimiento sobre peces}

Los peces constituyen un recurso importante e histórico para el pueblo q'eqchi' en Santa Lucía Lachuá, ya que las personas realizan actividades relacionadas con la pesca artesanal en los ríos circundantes como el Chixoy, Icbolay, Tzetoc y otros (Monzón, 1999). En la ecorregión, la riqueza de peces sobrepasa las 35 especies, de las cuales, al menos 20 son utilizadas para consumo, (Granados-Dieseldorff et al., 2012; USAC, 
2004). Los entrevistados reconocieron alimentarse de al menos 11 especies de peces, resaltando su valor alimenticio para la comunidad y como fuente de ingreso económico al venderlos en las localidades aledañas. Las especies mojarra paleta ( $V$. melanura) y mojarra colorada $(V$. bifasciata) fueron las que presentaron un mayor IVU, por ser alimento y comercio a la vez (Figura 3). Otros usos como ornamento o simbólicos no fueron mencionados, probablemente debido a que una vez consumida su carne, los derivados (esqueleto y escamas) son desechados.

Los hallazgos encontrados están sujetos a limitaciones de tiempo para entrevistar a más personas, lo cual se evidenció en la representatividad de la muestra ( $10.3 \%$ de la población) respecto a la población total de Santa Lucía Lachuá (Real instituto de Antropología de la Gran Bretaña e Irlanda, 1966), así como limitaciones en la entrevista, al delimitar las categorías de uso y conocimiento de fauna, y la formulación de preguntas abiertas que indaguen sobre el manejo comunitario de la fauna. Esto pudo influir en los patrones detectados de sistematización de conocimiento, por lo que se recomienda aumentar el tiempo de muestreo y la inclusión de factores que pueden influir en el conocimiento local, como la escolaridad y la relación de las personas con el PNLL.

En este estudio se observó que existe un conocimiento trasladado de una generación a otra, sobre aspectos y uso de la fauna local, lo cual está relacionado con la vida comunitaria, su cosmovisión q'eqchi' y la importancia que representa en su territorio.

A pesar de la existencia de una barrera cultural entre la etnia q'eqchi' y el mundo ladino occidental, la comprensión de sus conocimientos sobre la fauna permiten comprender las concepciones de usos y prácticas de la naturaleza. Sin embargo, la pérdida de este conocimiento local en Santa Lucía Lachuá podría aumentar si no se pone en práctica o se incentiva su transmisión. Esta es amenazada por las mismas instituciones educativas que no integran la realidad comunitaria a su sistema de aprendizaje, junto con los medios de información, así como la pobreza, desigualdad, abandono institucional, y otras actividades como la industrial y construcción que provocan la disminución de cobertura boscosa en los alrededores, la contaminación del recurso hídrico y pérdida de especies, modificando el entorno comunitario, donde además de la pérdida de diversidad biológica también se pierde la diversidad cultural debido a la degradación de la fuente principal de este conocimiento, la naturaleza. Por lo que es importante tomar en cuenta los conocimientos q'eqchi' en la definición de políticas locales que incluyan el sector educativo y de salud, el uso de tierras y actividades económicas, así como en la conservación de la diversidad biológica, considerando las comunidades adyacentes a áreas protegidas.

Por último, resaltamos la importancia de continuar los estudios etnozoológicos en la ecorregión Lachuá, asegurando que exista un proceso de investigación participativa, ética y que respete el diálogo de saberes, que permita fortalecer la relación entre la Escuela de Biología y los comunitarios de Santa Lucía Lachuá.

\section{Agradecimientos}

A la comunidad de Santa Lucía Lachuá por su apoyo en el trabajo de campo en especial a Ángel Xo, Edwin Xo, Juanita Caal, Aura Tox Caal, Hector Tox y Ramiro Tox (QEPD). A los profesores encargados del Curso Taller de Investigación Aplicada de la Universidad de San Carlos de Guatemala: Jorge Erwin López, Gustavo Ruano, Sergio Pérez, Claudio Méndez, a Dioné Méndez Barrios del Centro de Estudios en Salud de la Universidad del Valle de Guatemala, y a la Escuela de Biología por el desarrollo del curso taller en la comunidad.

\section{Referencias}

Avendaño Mendoza, C. E. (2001). Caracterización de la Avifauna del Parque Nacional Laguna Lachuá y su zona de influencia, Cobán, Alta Verapaz [Tesis de Licenciatura, Universidad de San Carlos de Guatemala]. https://biblioteca-farmacia.usac.edu. gt/Tesis/B121.pdf

Alves Barbosa, J. A., Asevedo Nobrega, V., \& da Nóbrega Alves, R. R. (2010). Aspectos da caça e comércio ilegal da avifauna silvestre por populações tradicionais do semi-árido paraibano. Revista de Biologia e Ciências da Terra, 10(2), 39-49.

Caballero Mariscal, D. (2013). Transculturación, sincretismo y pervivencia de la cultura entre los mayas- q'eqchi'es de Guatemala. Etnicex, (5), 65-82.

Caniago, I., \& Siebert, S. F. (1998). Medicinal plant ecology, knowledge and conservation in Kalimantan, Indonesia. Economic Botany, 52(3), 229250. https://doi.org/10.1007/BF02862141 
Consejo Nacional de Áreas Protegidas. (2003). Plan Maestro Parque Nacional Laguna Lachuá, Cobán, Alta Verapaz. https://conap.gob.gt/wp-content/uploads/2019/10/PM-PN-Laguna-Lachu\%C3\%A1.pdf

Consejo Nacional de Áreas Protegidas, Unión Internacional para la Conservación de la Naturaleza, Embajada de los Países Bajos \& Instituto Nacional de Bosques. (2004). Plan Maestro del Parque Nacional Laguna de Lachuá, Cobán, Alta Verapaz 2004-2009. Guatemala,

Consejo Nacional de Áreas Protegidas, Instituto Nacional de Bosques, \& Ministerio de Agricultura, Ganadería y Alimentación. (2011). Plan Maestro 2012-2016 del Parque Nacional Laguna Lachuá, Cobán, Alta Verapaz.

Cortés-Gregorio, I., Pascual-Ramos, E., MedinaTorres, S., Sandoval-Forero, E., Lara-Ponce, E., Piña-Ruíz, H., Martínez-Ruíz, R., Rojo-Martínez, G. E. et al. (2013). Etnozoología del pueblo mayo-yoreme en el norte de Sinaloa: Uso de vertebrados silvestres. Agricultura, Sociedad y Desarrollo, 10(3), 335-358.

De la Ossa-Lacayo, A., \& De la Ossa, J. (2012). Índice de valor de uso para fauna silvestre en la Región del San Jorge, Mojana Sucreña, Colombia. Revista Colombiana de Ciencias Animales, 4(2), 308-319.

Donato, L. M., Escobar, E. M., Escobar, P., Pazmiño, A., \& Ulloa, A. (Eds.) (2007). Mujeres indigenas, territorialidad y biodiversidad en el contexto latinoamericano. Universidad Nacional de Colombia-Fundación Natura de Colombia-Unión Mundial para la Naturaleza-UNODC-Oficina de las Naciones Unidas contra la Droga y el Delito.

dos Santos Rodrigues, A., (2009). Metodología de la investigación etnozoológica. En E. Costa, D. Santos \& M. Vargas (Coords). Manual de Etnozoología. Una guía teórico-práctica para investigar la interconexión del ser humano con los animales. Tundra Ediciones.

Estrada, A. C. (2006). Li Tzuultaq'a ut li ch'och'. Una visión de la tierra, el mundo y la identidad a través de la tradición oral Q'eqchi' de Guatemala. Estudios de Cultura Maya, 2, 149-163.

Escuela de Biología, Facultad de Ciencias Químicas y Farmacia, Universidad de San Carlos de Guate- mala. (2004). Ficha Informativa de los Humedales Ramsar (FIR). https://conap.gob.gt/wp-content/ uploads/2019/09/1623-Ecoregi\%C3\%B3n-Lachua.pdf

Flores, C. (2002). Apuntes sobre la dimensión cultural del conflicto armado entre los q'eqchi' de Guatemala. Desacatos, (10), 167-178.

García-Flores, A., Mojica-Pedraza, S., Barreto-Sánchez, S., Monroy-Ortiz, C., \& Monroy-Martínez, R. (2017). Estudio etnozoológico de las aves y mamíferos silvestres asociados a huertos frutícolas de Zacualpan de Amilpas, Morelos, México. Revista de Ciencias Ambientales (Trop J Environ Sci), 51(2), 110-132. https://doi.org/10.15359/ rca.51-2.6

Gómez-Baggethun, E. (2009). Perspectivas del conocimiento ecológico local ante el proceso de globalización. Papeles, 107, 57-67.

González, A. L. (2004). Caracterización etnobiológica de las actividades agrícolas fundamentales (Maíz y Cardamomo) y cinegética en tres comunidades Q'eqchi' es del área deinfluencia del Parque $\mathrm{Na}$ cional Laguna Lachuá, Cobán, Alta Verapaz [Tesis de licenciatura, Universidad de San Carlos de Guatemala]. https://biblioteca-farmacia.usac.edu. gt/Tesis/B141.pdf,

González-Bocanegra K., Romero-Berny, E., Escobar-Ocampo, M., \& García-Del Valle, Y. (2011). Aprovechamiento de fauna silvestre por comunidades rurales en los humedales de Catazajá - La Libertad, Chiapas, México. Ra Ximhai, 7(2), 219230. https://doi.org/10.35197/rx.07.02.2011.06. $\mathrm{kg}$

Granados-Dieseldorff, P., Christensen, M. F., \& Kihn-Pineda, P. H. (2012). Fishes from Lachuá Lake, Upper Usumacinta Basin, Guatemala. Check list, 8(1), 95-101. https://doi.org/10.15560/8.1.095

Grandía, L. (2018). Los restos: Renacimiento y resiliencia del pueblo Q'eqchi” en Petén. Ciencias Sociales y Humanidades, 5(1), 31-43. https://doi. org/10.36829/63CHS.v5i1.646

Guerra, R. M., Calmé, S., Gallina, T. S., \& Naranjo, P. E. (2010). Uso y manejo de la fauna silvestre en el norte de Mesoamérica. Secretaría de Educación, Gobierno del Estado de Veracruz, Xalapa, Veracruz. 
Gutiérrez-Santillán, T., Arellano-Méndez, L., \& Mora-Olivo, A. (2017). Etnozoología en México: Una revisión al estado del conocimiento. Revista Cientifica Multidisciplinaria De La Universidad De El Salvador-Revista Minerva, 1(1), 54-63.

Hatse, I., \& De Ceuster, P. (2001). Prácticas agrosilvestres $Q$ 'eqchi'es: Más allá del maíz y el frijol un aporte para la revalorización y el fortalecimiento de la agricultura tradicional q'eqchi'. Ak' Kutan.

Hermes, M. S. (2004). Abundancia relativa de jaguar (Panthera onca), puma (Puma concolor) y ocelote (Leopardus pardalis) en el Parque Nacional de Lachuá, Cobán, Alta Verapaz [Tesis de licenciatura, Universidad de San Carlos de Guatemala]. https://biblioteca-farmacia.usac.edu.gt/Tesis/ B140.pdf

Hermes, M. S., \& Rosales-Meda, M. (2011). Monitoreo Ecológico Participativo de la Riqueza y Distribución Espacial de mamíferos amenazados y en peligro de extinción en el Parque Nacional Laguna Lachuá y remanentes boscosos de su área de influencia, Alta Verapaz. Instituto de Investigaciones Químicas y Biológicas, Facultad de Ciencias Químicas y Farmacia, USAC (Inf-2011-23). Universidad de San Carlos de Guatemala, Dirección General de Investigación, Programa Universitario de Investigación en Recursos Naturales y Ambiente. http://digi.usac.edu.gt/bvirtual/informes/puirna/INF-2011-023.pdf

Hernández-Freeman, L. (2009). Concepción sociocultural de la gestión participativa de los grupos de trabajo comunitario integrado [Tesis de doctorado, Universidad de Oriente, Cuba]. https:// repositorio.uho.edu.cu/xmlui/bitstream/handle/ uho/2665/tes.pdf? sequence $=1 \&$ isAllowed $=y$

Hernández, S. (2004). Estudio etnoecológico del uso de vida silvestre y actividades de agricultura en dos comunidades del área de influencia del Parque Nacional Laguna Lachuá, Cobán, Alta Verapaz: Roq-ha' Purib'al y San Benito I [Tesis de licenciatura]. Universidad de San Carlos de Guatemala]. https://biblioteca-farmacia.usac.edu.gt/ Tesis/B144.pdf

Hernández-López, A., López-Alamilla, E., Rodríguez-Ramírez, A., y Aquino-Bravata, V. (2012). Diagnóstico del uso de la fauna silvestre en el área de protección de flora y fauna Cañón del Usumacinta, Tenosique Tabasco. Revista Ra Ximhai, 9(1), 1-13.
Hunn, E. (2011). Ethnozoology. En N. Anderson, D., Pearsall, E., Hunn \& N., Turner (Eds.). Ethnobiology (pp. 83-96). Wiley-Blackwell.

Koster, J., Bruno, O., \& Burns, J. L. (2016). Wisdom of the Elders? Ethnobiological Knowledge across the Lifespan. Current Anthropology, 57(1), 113121. https://doi.org/10.1086/684645

Ladio, A. H. (2020). La etnobiología en áreas rurales y su aporte a la lucha para desentrañar sesgos patriarcales. Ethnoscience, 5(1), 1-13. https://doi. org/10.22276/ethnoscientia.v5i1.298

López-Carrera, C., Zazueta-Angulo, X., \& Porras-Andujo, C. (2005). Notas sobre aprovechamiento del medio ambiente noroestense: Reconocimiento del terreno, inicio de la cacería. Revista Ra Ximhai, 1(1), 39-50.

Lugo-Morín, D. R. (2007). Aves de caza del grupo indígena E'ñepa de Guaniamo, Venezuela. Revista Científica y Técnica de Ecología y Medio Ambiente, 16(2), 86-97.

Marqués-Valdés, E. (2014). Acceso al agua y el saneamiento en la Ecorregión Lachuá, Guatemala. Estudio de caso: Diagnóstico general del sector en la comunidad Las Promesas - Nueve Cerros [Tesis de maestría, Universidad Politécnica de Madrid]. http://oa.upm.es/32416/1/TESIS_MASTER_ESTHER_MARQUES_VALDES_B.pdf

Millán, L., Arteaga, T. T., Moctezuma, S., Velasco, J. J., \& Arzate, J. C. (2016). Conocimiento ecológico tradicional de la biodiversidad de bosques en una comunidad matlatzinca, México. Ambiente y Desarrollo, 20(38), 111-123

Monroy, R., García, A., \& Pino, J. (2011). Etnozoología: Un Enfoque Binacional México - Colombia. Universidad Autónoma del Estado de Morelos.

Monzón, R. M. (1999). Estudio general de los recursos agua, suelo y del uso de la tierra del Parque Nacional Laguna Lachuá y su zona de influencia. Cobán, Alta Verapaz [Tesis de licenciatura, Universidad de San Carlos de Guatemala]. http://biblioteca.usac.edu.gt/tesis/01/01_1834.pdf

Morales, J. (1993). Caracterización etnozoológica de la Actividad de Cacería en la Comunidad de Uaxactún, Flores, El Petén [Tesis de licenciatura no publicada]. Universidad de San Carlos de Guatemala. 
Osorio, R. (1998). El cuestionario. La sociología en sus escenarios, (1), 1-15.

Otzen, T., \& Manterola, C. (2017). Técnicas de muestreo sobre una población a estudio. International Journal of Morphology, 35(1), 227-232. https:// doi.org/10.4067/S0717-95022017000100037

Pellecer, J., M., Morales, J. R., \& Pérez, S. G. (2019). Noteworthy records of the northern naked-tailed armadillo, Cabassous centralis (Cingulata: Chlamyphoridae), in Guatemala, Central America. Edentata, (20), 17-21. https://doi.org/10.2305/ iucn.ch.2019.edentata-20-1.3.

Pérez, E., \& Ojasti, J. (1996). La utilización de la fauna silvestre en la América Tropical y recomendaciones para su manejo sustentable en las sabanas. Ecotropicos, 9(2), 71-82.

Racero-Casarrubia, A., Vidal, C., Ruíz, O., y Ballesteros, J. (2008). Percepción y patrones de uso de la fauna silvestre por las comunidades indígenas Embera-Katíos en la cuenca del río San Jorge, zona amortiguadora del PNN-Paramillo. Revista de Estudios Sociales, 31, 118-131.

Real Instituto de Antropología de la Gran Bretaña e Irlanda. (1966). Guía de campo del investigador social. Unión Panamericana.

Rodas-Trejo, J., Ocampo-González, P., \& Coutiño-Hernández, P. (2014). Uso de los mamíferos silvestres en el municipio de Copainalá, región Zoque, Chiapas; México. Quehacer Científico en Chiapas, 9(1), 3-9.

Rojas, O., \& Avendaño, C. (2018). Relación entre áreas y variables ambientales espaciales con el atropellamiento y cruce de la fauna silvestre en la carretera de la Franja Transversal del Norte, Cobán, Guatemala. Ciencia, Tecnología y Salud, 5(2), 111-124.

Rosales, M., Hermes, M., \& Morales, J. (2010). Capítulo 1: Caracterización de la cacería de subsistencia en comunidades Maya-Q'eqchi' del área de influencia del Parque Nacional Laguna Lachuá, Guatemala. En R. M. Guerra, S. Calmé, T. S., Gallina \& P. E. NaranjoUso y manejo de la fauna silvestre en el norte de Mesoamérica (pp. 25-52). Secretaría de Educación, Gobierno del Estado de Veracruz, Xalapa, Veracruz.

Ruano, E., Méndez, D., \& Gaitán, C. (2019). Defaunación en el norte de Alta Verapaz: Posibles consecuencias en la diversidad de roedores y sus patógenos zoonóticos (Inf-2019-34). Universidad de San Carlos de Guatemala, Dirección General de Investigación, Programa Universitario de Investigación en Recursos Naturales y Ambiente. https://digi.usac.edu.gt/bvirtual/informes/puirna/ INF-2019-34.pdf

Russell, H. (2006). Métodos de Investigación en Antropología Abordajes cualitativos y cuantitativos. Alta Mira Press.

Santos-Fita, D., Argueta, A., Astorga-Domínguez, M. y Quiñonez-Martínez, M. (2012). La Etnozoología en México: La Producción Bibliográfica del Siglo XXI -2000-2011- . Etnobiología, 10(1), 4151 .

Sociedad Latinoamericana de Etnobiología -SOLAE-. (2016). Código de Etica. Etnobiología, 14(Suplemento 1), 5-30.

Stuart, S. N., Hoffmann, M., Chanson, J. S., Cox, N. A., Berridge, R. J., Ramani, P., \& Young, B. E. (2008). Threatened amphibians of the world. Lynx Editions

Tlapaya, L., y Gallina, S. (2010). Cacería de mamíferos medianos en cafetales del centro de Veracruz, México. Acta Zoológica Mexicana, 26(2), 259277. https://doi.org/10.21829/azm.2010.262698

Universidad de San Carlos de Guatemala. (2004). Ficha Informativa de los Humedales de Ramsar FIR, Eco-región Lachuá. Recuperado de: https:// rsis.ramsar.org/RISapp/files/RISrep/GT1623RIS. pdf

Vázquez-García, V. (2007). Gender, ethnicity, and economic status in plant management: uncultivated edible plants among the Nahuas and Popolucas of Veracruz, Mexico. Agriculture and Human Values, 25, 65-77. https://doi.org/10.1007/ s10460-007-9093-x

Vela, F. (2001). Un acto metodológico básico de la investigación social: La entrevista cualitativa. En Tarrés, M.(Coord.). Observar, escuchar y comprender sobre la tradición cualitativa en la investigación social. México: Editorial Porrúa.

Viu, A., Viu, M. \& Campos, L. (2010). Etnobotânica: Uma questão de gênero? Revista Brasileira de Agroecologia, 5(1), 138-147.

Wendt, T. (1987). Selvas de Uxpanapa, Veracruz-Oaxaca, México: Evidencia de Refugios Florísticos 
Cenozoicos. Anales Instituto de Biología. UNAM Serie Botánica, 58, 29-54.

Wilson, R. (1999). Resurgimiento Maya en Guatemala (Experiencias Q'eqchies). Centro de investigaciones regionales de Mesoamérica. 